\title{
Air Distribution Simulation of Thermal Environment With Airpack Software in Ship Cabins
}

\author{
Hong-Min Liu, Ran-Ran Xu \\ Merchant Marine College of Shanghai Maritime University, Shanghai, China \\ E-mail: lhmnjj@126.com, 18217060651@163.com
}

\begin{abstract}
The environment quality of ship cabins will directly affect the crew work efficient and health. In order to analyze the effect of different air-supply types on ship's cabin environment, one rest room of a ship was selected as the research object. Based on the computational fluid dynamics and numerical heat transfer theory, numerical simulation was carried out for different air-supply types with the software AIRPAK. Through the comparative analysis of temperature field, PMV(Predicted Mean Vote) field, PPD(Percentage of Predicted Dissatisfaction) field, airflow distribution of up-supply and down-return air-conditioning system is more even and this system can provide better thermal comfort and meet the comfort requirements of the crew in the cabin.
\end{abstract} $P M V$

Keywords-airpack software; thermal comfort; air distribution;

\section{INTRODUCTION}

At the beginning of thermal comfort research, research methods were confined to subjective questionnaire combined with experiment. Questionnaire research needed on-spot investigation and needed to measure multiple thermal parameters. With the development of computer technology, thermal comfort research integrating CFD (Computer Fluid Dynamics) was widely applied and simulation could forecast the research results at least cost. CFD simulation was convenient and fast to analyze air distribution under different thermal environment, and optimize human body thermal comfort under different thermal environment. The numerical simulation of the air distribution in inland building is more ${ }^{[1-5]}$, while there is less research on the air distribution in ship cabins. In this paper, a crew rest room is selected as the research object. Through the CFD numerical simulation, air distribution with different air-supply types is analyzed and optimized.

\section{AIRPAK SOFTWARE}

Airpak is an analysis software of professional artificial environment system and is developed on the basis of a software package in CFD technology. Airpak provides powerful numerical report and can simulate the indoor temperature field and humidity field, velocity field, pollutants concentration field, air age, PMV, PPD field, etc under different supply air distribution forms of air conditioning system.

Airpak software has the following features:

1) Modeling fast: Airpak is based on the "object" of modeling; the "object" includes room, the human body block, fan, vents, wall and partition, heat load source and so on. Airpak also provides the interface with CAD software. CAD geometry can be imported by IGES and DXF format.

2) Automatic meshing functions: Airpak have automated grid generation ability. It supports tetrahedron, hexahedron, and hybrid grid, thus it can generates high quality grids of the model. Airpak can also check grids powerfully and it can distinguish poor quality grids.

3) Unstructured grid technology: it can simulate approximately bodies with complicated shape geometry, greatly reducing the number of grid and improving the model's accuracy.

4) The strong ability of modeling: It can set up the models of forced convection, natural convection, mixed convection; heat conduction, heat transfer, thermal radiation mode; laminar flow, turbulent flow, the steady state and transient problem.

5) Powerful calculating function: It has a FLUENT solver, the world's most powerful CFD (computational fluid dynamics) solver. Finite Volume Method (Finite Volume Method) and the solver of structured and unstructured grid can calculate and implement the UNIX or NT network in parallel.

6) Powerful visual processing: it can fully integrate processing environment and provide visuable velocity vector chart and contour plot of temperature, humidity, pressure and concentration etc.

\section{SHIP CABIN MODEL}

The crew rest room is selected as the simulation object. The room size is $10 \mathrm{~m}$ long, $5 \mathrm{~m}$ wide and $2.1 \mathrm{~m}$ high. There are two doors ( $1 \mathrm{~m} * 2 \mathrm{~m}$, closed), a table, four fluorescent lamp, five sofas, a TV, 10 resting people .Physical model is shown in Fig.1. 


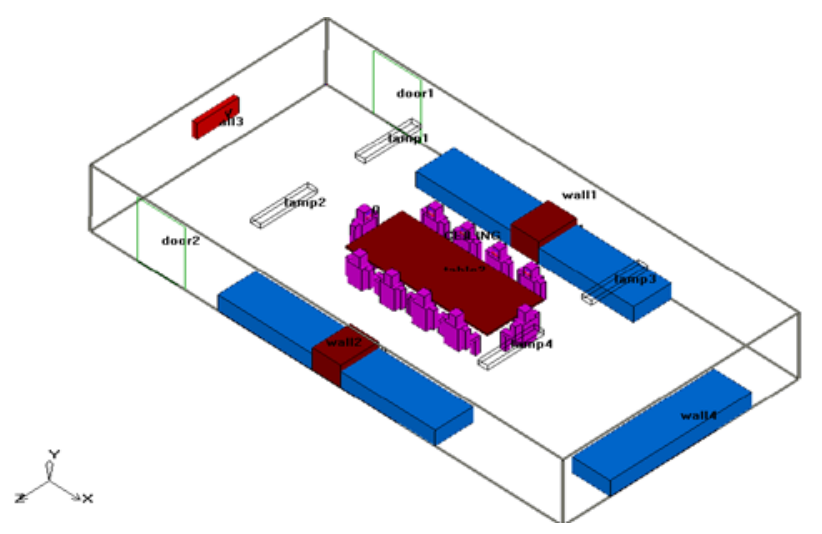

Figure 1. Physical model of rest room.

The actual indoor air flow involves the gas flow, heat transfer, mass transfer, etc. In air conditioning air distribution

Quality equation

$$
\frac{\partial V_{i}}{\partial \boldsymbol{x}_{i}}=\mathbf{O}
$$

Momentum equation

$$
\frac{\partial\left(\rho V_{i}\right)}{\partial t}+\frac{\partial \rho V_{i} V_{j}}{\partial x_{j}}=-\frac{\partial p}{\partial x_{i}}+\frac{\partial}{\partial x_{j}}\left[\mu_{e f f}\left(\frac{\partial V_{i}}{\partial x_{j}}+\frac{\partial V_{j}}{\partial x_{i}}\right)\right]+\rho \beta\left(T_{0}-T\right) g_{i}
$$

Energy equation

$$
\frac{\partial \rho T}{\partial t}+\frac{\partial \rho V_{j} T}{\partial x_{j}}=\frac{\partial}{\partial x_{j}}\left(\Gamma_{T, \text { eff }} \frac{\partial T}{\partial x_{j}}\right)+\frac{q}{C_{p}}
$$

Mass transfer equation

$$
\frac{\partial \rho C}{\partial t}+\frac{\partial \rho V_{j} C}{\partial x_{j}}=\frac{\partial}{\partial x_{j}}\left(\Gamma_{C, \text { eff }} \frac{\partial C}{\partial x_{j}}\right)+S_{C}
$$

Where,

$\mu_{e f f}=\mu+\mu_{t}, \Gamma_{e f f}=\frac{\mu_{e f f}}{\operatorname{Pr}_{e f f}}, \mu_{t}=0.03874 \rho v l ;$ $V_{i}$--the speed o $x_{i}$ direction (i=1, 2, 3), m/s; t--time, s; $\rho$--air density, $\mathrm{kg} / \mathrm{m}^{3} ; p$--air pressure, Pa; $\mu$--air laminar flow dynamic viscosity, Pa.s; $\mu_{t}$--air turbulence kinetic viscosity, Pa.s; $\beta$--Air thermal expansion coefficient; $g_{i}$--gravity acceleration of the $\mathrm{x}$ direction, $\mathrm{m} / \mathrm{s}^{2} ; \quad T$--air temperature, ${ }^{\circ} \mathrm{C} ; \Gamma_{T, \text { eff }}$--the diffusion coefficient of temperature , $\mathrm{kg} /(\mathrm{m} . \mathrm{s}) ; \quad q$--the heat source, $\mathrm{W} / \mathrm{m}^{3} ; \quad C_{p} \quad$--specific heat capacity, $\mathrm{J} /\left(\mathrm{Kg} .{ }^{\circ} \mathrm{C}\right)$; $\mathrm{Pr}_{\text {eff }}$--equivalent prandtl number, $\mathrm{Pr}_{\text {eff }}=0.9 ; C$--the volume fraction of components; $\Gamma_{C, \text { eff }}$--the diffusion coefficient of components, $\mathrm{kg} /(\mathrm{m} . \mathrm{s}) ; S_{C}$--components of the source term; $v$--mean velocity, $\mathrm{m} / \mathrm{s} ; l$--away from the wall in recent distance, $\mathrm{m}$.
In the actual situation, the air distribution of the cabin will be affected by many factors. In order to simplify the problem, assumptions are made as follows.

(1)Air in the cabin is incompressible fluid, at low speed and in line with the Boussinesq hypothesis ${ }^{[8]}$; (2) Air flow is steady and turbulent; (3) The viscous dissipation of the fluid is negligible; (4) Radiation heat transfer is ignored; (5) This paper is to investigate cabin internal flow field under steady state conditions and doesn't consider the effect of air leakage.

Two air supply schemes are designed and illustrated in Fig. 2, and volume of supply air is $1920 \mathrm{~m}^{3} / \mathrm{h}$. Supply air temperature is $22{ }^{\circ} \mathrm{C}$ and air velocity is $1 \mathrm{~m} / \mathrm{s}$. Outlet let pressure is environmental pressures. The temperature difference between indoor and outdoor for wall with the door is $2^{\circ} \mathrm{C}$, heat transfer coefficient is $1.1 \mathrm{~W} /\left(\mathrm{m}^{2} \cdot \mathrm{k}\right)$. Wall 3 and wall 4 are adiabatic. Heat transfer coefficient of ceiling is $0.8 \mathrm{~W} /\left(\mathrm{m}^{2} . \mathrm{k}\right)$. The floor is adiabatic. Internal heat load: for a person is $75 \mathrm{~W}$, the power of fluorescent lamp is $40 \mathrm{~W}$ and $T V$ is $210 \mathrm{~W}$.

Air temperature, PMV and PPD are chosen as indexes to evaluate the performance of different air-conditioning systems. 


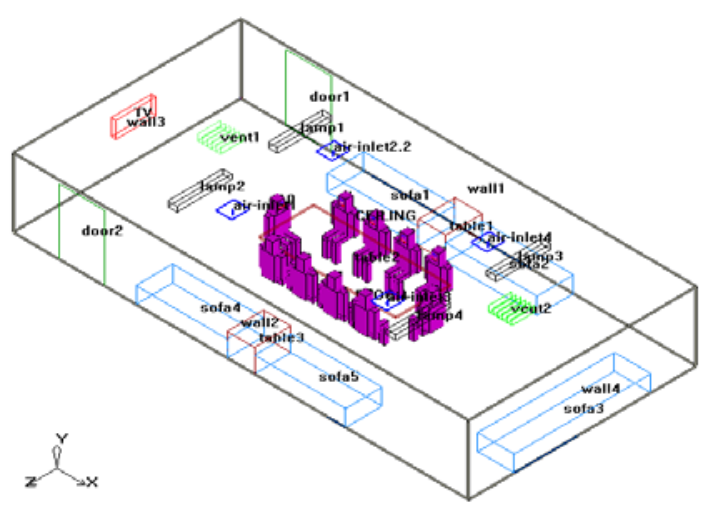

(a)The model of the up-supply and up-return system, scheme(a)

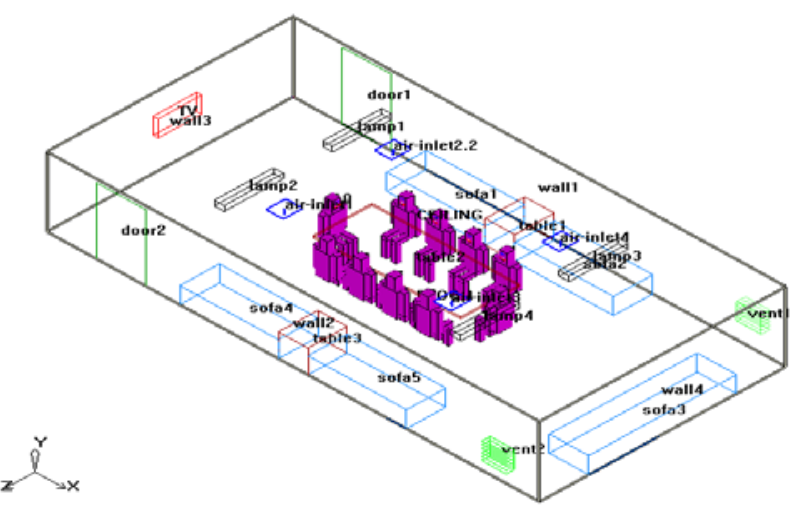

(b)The model of the up-supply and down- return system, scheme(b)

Figure 2. The physical model of different air systems.

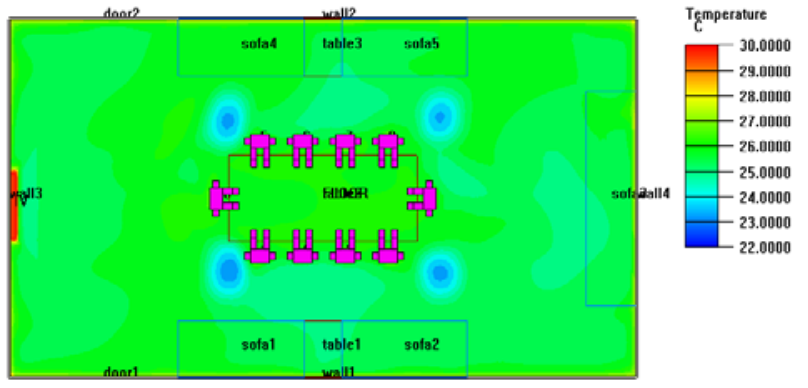

(a) The up-supply and up-return system

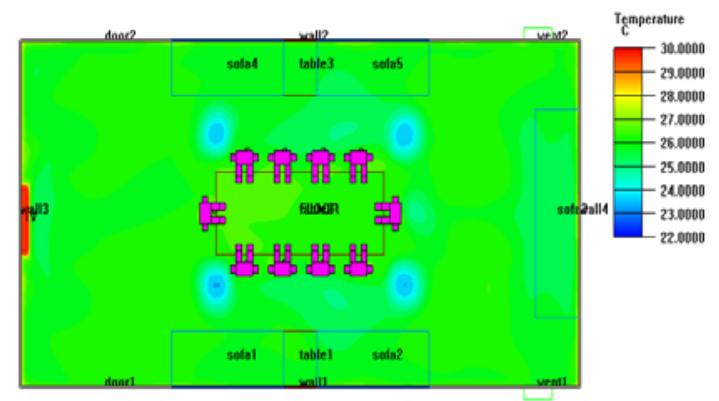

(b) The up-supply and down-return system

Figure 3. The temperature field distribution of $y=1.4 \mathrm{~m}$ section.

Simulation result analysis

Fig. 3 shows the temperature distribution of $y=1.4 \mathrm{~m}$ (human head) section for two schemes. From Fig. 3(a), air temperature field is low and air distribution is uneven for the up-supply and up-return system. Compared to Fig. 3(a), distribution of temperature field is more uniform, and the temperature of the room is between 24 and $27^{\circ} \mathrm{C}$ in Fig. 3(b) of the up-supply and down-return system. Furthermore, temperature stratification is more likely to happen in the up-supply and up-return system. Therefore, the scheme (b) is much better.

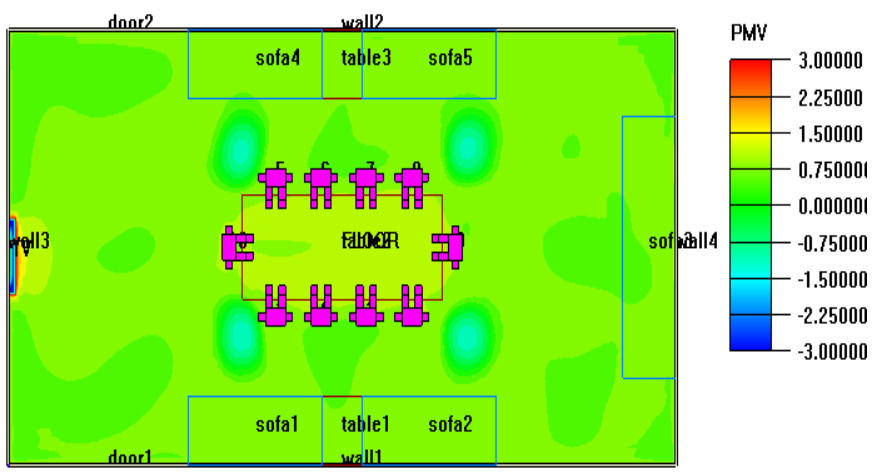

(a) The up-supply and up-return system

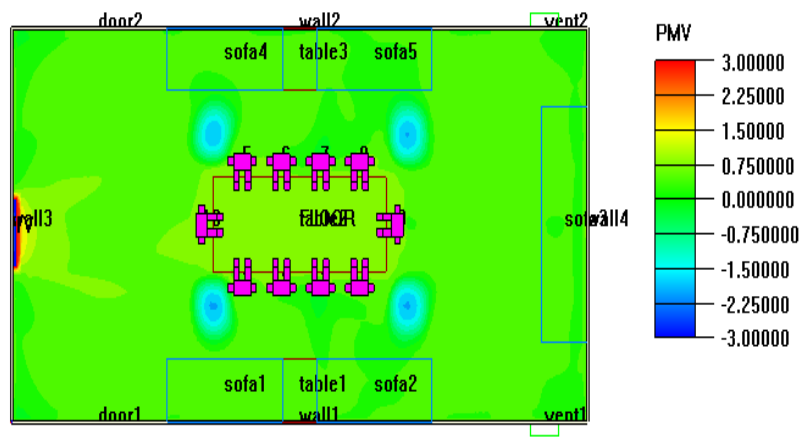

(b) The up-supply and down- return system

Figure 4. The PMV distribution of $\mathrm{y}=1.4 \mathrm{~m}$ section. 
Similarly, $y=1.4 \mathrm{~m}$ (human head) section of PMV distribution is analyzed. In up-supply and up-return system shown in Fig. 4(a), the PMV value of the human head is around 1.5 and people feel uncomfortable hot. In contrast,

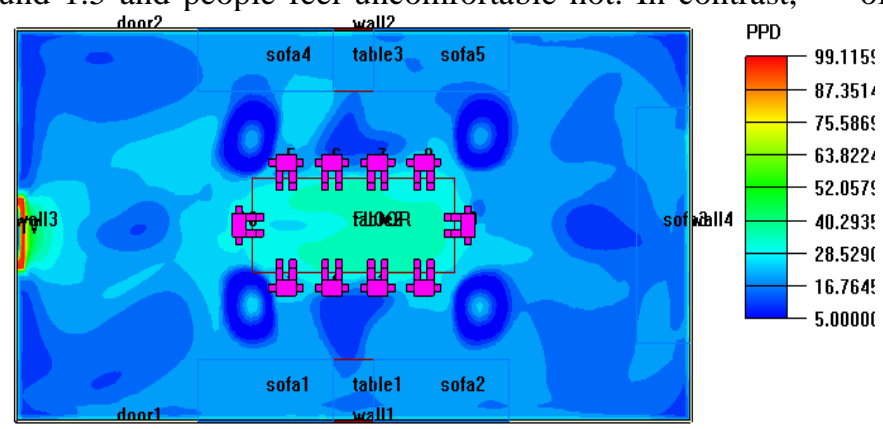

(a) The up-supply and up-return system the PMV value of the human head is nearly 0.75 in Fig. 4(b) and occupants feel comfortable. About $80 \%$ of occupants would feel satisfied in such a pleasant environment. In view of PMV, the scheme (b) also definitely wins.

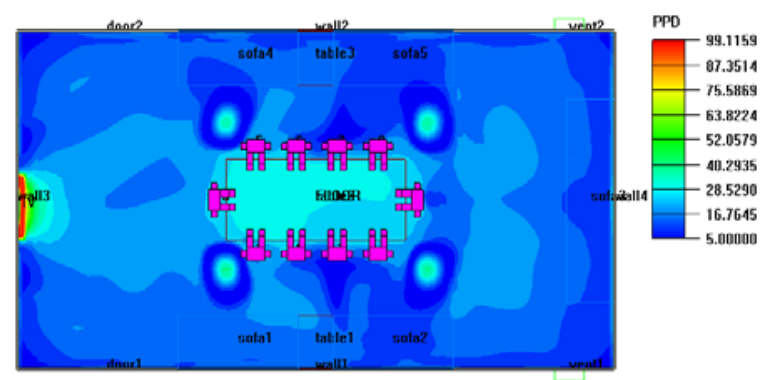

(b) The up-supply and down- return system

Figure 5. The PPD distribution of $y=1.4 \mathrm{~m}$ section.

Fig. 5 illustrates PPD distribution of the section of $y=1.4 m$. From Fig. 5 (a) for scheme (a), the PPD value around human head is $40 \%$, much higher than the accepted level. While PPD value at the same zone is $25 \%$, nearly meeting the thermal comfort requirement. Obviously, scheme (b) would be preferred by the occupants.

\section{CONCLUSIONS}

This paper set up the mathematical model of the cabin with the software Airpack and numerical simulation was carried out for two different air-supply types, the up-supply and up-return system and the up-supply and down-return system. The effect of two schemes was compared. Through the comparative analysis, air temperature and PMV distribution are more uniform in the up-supply and down-return system. And also there is no air stratification phenomenon. The PMV value is between -0.75 and 0.75 and PPD approaches to $25 \%$, meeting the requirement of thermal comfort. So it is advised to choose the scheme (b) for the crew rest cabin.

\section{ACKNOWLEDGEMENT}

This research was financially supported by the National Natural Science Foundation (No. 51406112) and Shanghai Municipal Education Commission Foundation (N0.
14YZ116)

\section{REFERENCES}

[1] Wei Yang, Nyuk Hien Wong, Yaolin Lin Thermal Comfort in High-rise Urban Environments in Singapore. Procedia Engineering. 121(2015) 2125-2131

[2] S.A. Nada, H.M. El-Batsh, H.F. Elattar, N.M. Ali.CFD investigation of airflow pattern, temperature distribution and thermal comfort of UFAD system for theater buildings applications.Journal of Building Engineering. 6(2016) 274-300.

[3] Catalin Teodosiu, Viorel Ilie, Raluca Teodosiu. Numerical Prediction of Thermal Comfort and Condensation Risk in a Ventilated Office, Equipped with a Cooling Ceiling.Energy Procedia. 85(2016) 550-558.

[4] Slah Driss, Zied Driss, Imen Kallel Kammoun.Computational study and experimental validation of the heat ventilation in a living room with a solar patio system.Energy and Buildings. 119(2016) 28-40.

[5] He Li hu, Luo Zhuo ying, Kuang Jing yu,etc. Numerical Simulation of Different of Air-condition Conference Rooms.Energy Conservation Technology. 3(2012)17-21. (in Chinese)

[6] Chen Qingyan, Xu Weiran.A zero-equation turbulence model for indoor air flow simulation. Energy and Building. 28(1998)137-144.

[7] Liu liang, Sun Li ying, etc. Numerical Simulation of Several Air Distributor Forms on Ship's Conference Air Environment. Fluid Machinery. 41(2013)73-77. (in Chinese)

[8] Cheng Dong mei. Research on Numerical Simulation of Airflow Organization of Ship Living Cabin [D].Harbin Engineering University, 2007. (in Chinese) 